\title{
Mantle Cell Lymphoma: Updates in the Management of Relapsed/Refractory Disease
}

\author{
Presented by Mazyar Shadman, MD, MPH
}

\begin{abstract}
Mantle cell lymphoma remains incurable despite recent treatment advances, and most patients experience relapsed/refractory disease. BTK inhibitors are the preferred choice in the relapsed setting, especially in patients with early relapse. For patients with high-risk features such as TP53 mutation, early referral for CAR T-cell therapy should be considered, even in those with stable disease on a BTK inhibitor. Patients without high-risk features may be monitored and initiate CAR T-cell therapy after clinical disease progression. CAR T-cell therapy is an effective treatment with high rate of complete remissions. For patients who do not achieve a complete remission 3 months after CAR-T therapy, bridging therapy with chemotherapy or targeted therapy agents and referral for allogeneic transplant are recommended.
\end{abstract}

J Natl Compr Canc Netw 2021;19(11.5):1331-1333 doi: $10.6004 /$ jnccn.2021.5110

Despite advancements in treatment, mantle cell lymphoma (MCL) is still considered incurable, and most patients experience relapsed/refractory (R/R) disease. However, according to Mazyar Shadman, MD, MPH, Associate Professor, Clinical Research Division, Fred Hutchinson Cancer Research Center/University of Washington, the goal of treatment may be changing from palliation to more prolonged remission and possibly a future cure. At the NCCN 2021 Virtual Congress: Hematologic Malignancies, Dr. Shadman discussed effective treatment approaches and modalities in the current armamentarium, as well as novel treatment modalities that could change the goals of care.

\section{Frontline Setting}

As Dr. Shadman explained, for incurable diseases with limited therapeutic options, it is critical to identify the optimal sequence in order to provide the best clinical outcome for patients. It is also important for patients to be aware of the "big picture plan." "When we discuss treatment options with patients, we explain that multiple relapses are expected and that treatment options remain limited, even now," said Dr. Shadman. "The goal of therapy, especially at the time of relapse, is to delay disease progression and to palliate symptoms."

Most patients will receive some combination of chemotherapy backbone plus a CD20 antibody in the frontline setting, although, according to Dr. Shadman, the choice of chemotherapy is still an area of controversy. Depending on treatment strategy, patient comorbidities, and patient preferences, some patients will also receive high-dose therapy followed by autologous stem cell transplantation as consolidation, and maintenance therapies with rituximab, which has been shown to provide an overall survival benefit. Other patients may undergo maintenance rituximab after finishing chemoimmunotherapy, said Dr. Shadman.

\section{BTK Inhibitors}

As in the NCCN Clinical Practice Guidelines in Oncology (NCCN Guidelines) for B-Cell Lymphomas, BTK inhibitors are the preferred treatment for patients with $\mathrm{R} / \mathrm{R}$ MCL. ${ }^{1}$ According to Dr. Shadman, treatment of B-cell lymphomas has "drastically changed" with the introduction of the first-in-class BTK inhibitor ibrutinib. Despite a safety profile that sometimes leads to treatment discontinuation, ibrutinib remains an effective treatment choice, he said, and noted that adverse effects include atrial fibrillation and bleeding.

In addition to ibrutinib, 2 second-generation BTK inhibitors have been FDA-approved for treatment in MCL: zanubrutinib and acalabrutinib. Although there have been no head-to-head studies in MCL, said Dr. Shadman, randomized trials have shown an improved safety profile in in chronic lymphocytic leukemia and Waldenström macroglobulinemia with these second-generation BTK inhibitors compared with ibrutinib (Table 1$).^{2-6}$ However, there are no data indicating superior efficacy of second-generation BTKs in MCL. "BTK inhibitors remain a great treatment option, but they can't be a destination therapy," he continued. "Most patients will have a great but limited response." 
Table 1. BTK Inhibitors in R/R MCL

\begin{tabular}{|c|c|c|c|c|c|c|}
\hline & $\mathbf{N}$ & $\begin{array}{l}\text { Median Follow-Up } \\
\text { (mo) }\end{array}$ & $\begin{array}{l}\text { ORR } \\
(\%)\end{array}$ & $\begin{array}{l}\text { CR } \\
(\%)\end{array}$ & $\begin{array}{c}\text { TTF/PFS } \\
\text { (mo) }\end{array}$ & os \\
\hline Ibrutinib $^{2}$ & 370 & 42 & 70 & $27^{\mathrm{a}}$ & 12.5 & $26.7 \mathrm{mo}$ \\
\hline Ibrutinib + rituximab ${ }^{3}$ & 50 & 48 & 88 & 58 & 43 & $3-y: 69 \%$ \\
\hline Acalabrutinib ${ }^{4,5}$ & 124 & 24 & 80 & 40 & 15 & NA \\
\hline Zanubrutinibs & 86 & 36 & 84 & 78 & $\begin{array}{c}36 \\
\text { PFS: } 48 \%\end{array}$ & $\begin{array}{l}\text { 36-mo } \\
\text { PFS: } 75 \%\end{array}$ \\
\hline
\end{tabular}

Abbreviations: $\mathrm{CR}$, complete remission; $\mathrm{MCL}$, mantle cell lymphoma; NA, not available; ORR, overall response rate; OS, overall survival; PFS, progression-free survival; R/R, relapsed/refractory; TTF, time to treatment failure.

${ }^{a}$ Response assessment by CT.

Dr. Shadman reported that remissions in MCL shorten over time and with subsequent lines of therapy. A study by investigators at Memorial Sloan Kettering Cancer Center found a high correlation between progression-free and overall survival, which indicated waning efficacy and durability of response as treatment progresses. ${ }^{8}$ Additional observational studies have identified a benefit in progression-free and overall survival in patients who receive ibrutinib after first relapse. ${ }^{2}$ In later relapse, however, the outcomes between treatment regimens appear to be more similar. ${ }^{9}$

"Using BTK inhibitors early clearly showed a benefit in patients with R/R disease," said Dr. Shadman. "For high-risk patients who experience early relapse in the first-line setting (within 12-24 months), there is even more reason to use BTK inhibitors."

\section{Options Beyond BTK Inhibitors}

There are several other options beyond BTK inhibitor treatment in the R/R setting of MCL. Lenalidomide + rituximab is an established combination, and bortezomib can be used both as monotherapy or in combination with rituximab/dexamethasone (Table 2). ${ }^{10-12}$ "Using these drugs in the first relapsed setting is not preferred, but in patients with late relapse, if there is interest in time-limited therapy, giving chemoimmunotherapy or these novel agents is an option," said Dr. Shadman. "BTK inhibitors have been shown to be superior against other targeted agents in early relapse and in patients with no lines of prior therapy."

According to Dr. Shadman, selecting treatment after ibrutinib is challenging in both chronic lymphocytic leukemia and MCL, whether due to intolerance or disease progression. "In patients who have experienced disease progression on ibrutinib or other BTK inhibitors, we have consistently seen extremely poor outcomes," said Dr. Shadman. "This is an area of unmet need."

\section{CAR T-Cell Therapy}

As Dr. Shadman explained, transitioning patients to CAR T-cell therapy after disease progression on BTK inhibitors is a clinical art that requires bridging therapies to stabilize the disease.

A study of rituximab + bendamustine + cytarabine demonstrated a duration of response of $<1$ year in $61 \%$ of high-risk patients after BTK inhibitor therapy. ${ }^{13}$ According to Dr. Shadman, this combination regimen can be used to temporarily maintain stable disease (before CAR T-cell therapy), but it is not a destination therapy.

Approval of the CAR T-cell therapy brexucabtagene autoleucel for MCL was based on results of the ZUMA-2 study, ${ }^{14}$ which showed durable remissions in a majority of patients with R/R disease. Nearly $50 \%$ of patients in this high-risk population had prior autologous stem cell transplant, $>80 \%$ had received $>3$ prior lines of therapy, and $100 \%$ had prior therapy with BTK inhibitors, but the objective response rate was $93 \%$, with $67 \%$ of patients achieving a complete response. "We still need longer follow-up, but these data show an impressive initial response and continued remissions," said Dr. Shadman, who noted that a benefit was observed in all subgroups.

Nevertheless, the therapy also led to serious adverse effects, including cytokine-release syndrome (CRS) and neurologic toxicities. Approximately $30 \%$ of

Table 2. Other Agents/Combinations for Relapsed MCL

\begin{tabular}{|c|c|c|c|c|c|}
\hline & $\mathbf{N}$ & $\begin{array}{l}\text { Follow-Up } \\
\quad(\mathrm{mo})\end{array}$ & $\begin{array}{l}\text { ORR } \\
(\%)\end{array}$ & $\begin{array}{l}\text { CR } \\
(\%)\end{array}$ & $\begin{array}{l}\text { PFS } \\
\text { (mo) }\end{array}$ \\
\hline Lenalidomide + rituximab $^{10}$ & 52 & 23 & 57 & 36 & 11 \\
\hline Bortezomib + rituximab + dexamethasone ${ }^{12}$ & 16 & ? & 81 & 44 & 12 \\
\hline
\end{tabular}

Abbreviations: $C R$, complete remission; $M C L$, mantle cell lymphoma; ORR, overall response rate; PFS, progression-free survival; R/R, relapsed/refractory. 
patients experienced high-grade neurologic toxicities, and $15 \%$ had grade $\geq 3 \mathrm{CRS}$ and required intensive care with vasopressors. "Cellular immunotherapy using CAR T-cell therapy represents a very different approach that does not follow the classic risk factors of chemoimmunotherapy or even targeted drugs," said Dr. Shadman. "CRS and neurologic toxicities are side effects that need to be discussed with patients when undergoing CAR T-cell therapy, but this is still an extremely effective treatment."

According to Dr. Shadman, there remains no standard approach for the timing of referral for CAR T-cell therapy in patients treated with a BTK inhibitor. Some oncologists advocate for referral to CAR T-cell therapy while a patient is still on BTK inhibition and before disease progression, he said.

\section{Promising Therapeutic Agents}

Finally, new investigational agents are showing promise in MCL. Pirtobrutinib (LOXO-305), a noncovalent irreversible BTK inhibitor and a third-generation drug, is highly selective for BTK, said Dr. Shadman. He noted that data from the initial publication suggest a very favorable safety profile with pirtobrutinib. ${ }^{15}$ "The response rate in all patients with MCL, including those who with prior exposure to BTK inhibitors, is impressive [with pirtobrutinib], and these responses are durable," he added. "This drug is not yet FDA-approved, but studies are ongoing. Hopefully, this will soon be an option for our patients."

Dr. Shadman noted that pirtobrutinib may even be used in patients who experience disease progression after a first- or second-generation BTK inhibitor.

Although pirtobrutinib has not yet been approved for MCL, there are also data to support venetoclax both as monotherapy and in combination with ibrutinib. ${ }^{16}$ "This combination remains investigational, but in clinical practice, we've seen high efficacy with venetoclax + ibrutinib in patients after BTK inhibition," said Dr. Shadman.

Disclosures: Dr. Shadman has disclosed receiving consulting fees from AbbVie, Inc., Adaptimmune, Adaptive Biotechnologies, AstraZeneca Pharmaceuticals LP, Atara Biotherapeutics, BeiGene, Bristol-Myers Squibb Company, Eli Lilly and Company, Epizyme, Genentech, Inc., Innate Pharma, Kite Pharma, MorphoSys AG, Mustang Bio, Pharmacyclics, Sound Biologics, and TG Therapeutics; and receiving grant/research support from AbbVie, Inc., AstraZeneca Pharmaceuticals LP, Atara Biotherapeutics, BeiGene, Bristol-Myers Squibb Company, Celgene Corporation, Genentech, Inc. GenMab, Mustang Bio, Pharmacyclics, Sunesis Pharmaceuticals, Inc., and TG Therapeutics.

Correspondence: Mazyar Shadman, MD, MPH, Fred Hutchinson Cancer Research Center/University of Washington, 1100 Fairview Avenue North, D5-396, Seattle, WA 98109. Email: mshadman@fredhutch.org

\section{References}

1. Zelenetz AD, Gordon LI, Abramson JS, et al. NCCN Clinical Practice Guidelines in Oncology for B-Cell Lymphomas. Version 4.2021. Accessed September 1, 2021. To view the most recent version, visit NCCN.org

2. Rule S, Dreyling M, Goy A, et al. Ibrutinib for the treatment of relapsed/ refractory mantle cell lymphoma: extended 3.5-year follow up from a pooled analysis. Haematologica 2019;104:e211-214.

3. Jain $\mathrm{P}$, Romaguera J, Srour SA, et al. Four-year follow-up of a single arm, phase II clinical trial of ibrutinib with rituximab (IR) in patients with relapsed/refractory mantle cell lymphoma (MCL). Br J Haematol 2018; 182:404-411.

4. Wang $M$, Rule S, Zinzani $P L$, et al. Acalabrutinib in relapsed or refractory mantle cell lymphoma (ACE-LY-004): a single-arm, multicentre, phase 2 trial. Lancet 2018;391:659-667.

5. Wang M, Rule S, Zinzani PL, et al. Durable response with single-agent acalabrutinib in patients with relapsed or refractory mantle cell lymphoma. Leukemia 2019;33:2762-2766.

6. Song Y, Zhou K, Zou D, et al. ZANUBRUTINIB (zanu) in patients (pts) with relapsed/refractory (R/R) mantle cell lymphoma (MCL): long-term efficacy and safety results from a phase 2 study [abstract]. Presented at the European Hematology Association 2021 Virtual Congress; June 9-17 2021. Abstract EP789.

7. Kaptein A, de Bruin G, Emmelot-van Hoek M, et al. Potency and selectivity of BTK inhibitors in clinical development for B-cell malignancies [abstract]. Blood 2018;132(Suppl 1):Abstract 1871.

8. Kumar A, Sha F, Toure A, et al. Patterns of survival in patients with recurrent mantle cell lymphoma in the modern era: progressive shortening in

response duration and survival after each relapse. Blood Cancer J 2019; 9:50.

9. Visco C, Di Rocco A, Evangelista A, et al. Outcomes in first relapsedrefractory younger patients with mantle cell lymphoma: results from the MANTLE-FIRST study. Leukemia 2021;35:787-795.

10. Wang $M$, Fayad L, Wagner-Bartak $N$, et al. Lenalidomide in combination with rituximab for patients with relapsed or refractory mantle-cell lymphoma: a phase 1/2 clinical trial. Lancet Oncol 2012;13:716-723.

11. Fisher RI, Bernstein SH, Kahl BS, et al. Multicenter phase II study of bortezomib in patients with relapsed or refractory mantle cell lymphoma. J Clin Oncol 2006;24:4867-4874.

12. Lamm W, Kaufmann H, Raderer M, et al. Bortezomib combined with rituximab and dexamethasone is an active regimen for patients with relapsed and chemotherapy-refractory mantle cell lymphoma. Haematologica 2011;96:1008-1014.

13. McCulloch R, Visco C, Eyre TA, et al. Efficacy of R-BAC in relapsed, refractory mantle cell lymphoma post BTK inhibitor therapy. $\mathrm{Br} \mathrm{J}$ Haematol 2020;189:684-688.

14. Wang M, Munoz J, Goy A, et al. KTE-X19 CAR T-cell therapy in relapsed or refractory mantle-cell lymphoma. N Engl J Med 2020;382:1331-1342.

15. Mato AR, Shah NN, Jurczak W, et al. Pirtobrutinib in relapsed or refractory B-cell malignancies (BRUIN): a phase 1/2 study. Lancet 2021;397: 892-901.

16. Tam CS, Anderson MA, Pott C, et al. Ibrutinib plus venetoclax for the treatment of mantle-cell lymphoma. N Engl J Med 2018;378:1211-1223. 